\title{
DETERMINED EXOELECTRON EMISSION EFFECT IN DOPED TGS
}

\author{
G. I. ROSENMAN, $\uparrow$ M. S. TSEDRIK, \\ and YU. L. CHEPELEV† \\ †USSR 620032 Sverdlovsk, Urals Institute of Forestry \\ †USSR 220809 Minsk, Sovetskaya 18, Minsk State Pedagogic Institute
}

(Received June 13, 1989; in final form November 30, 1989)

\begin{abstract}
The exoelectron emission from ADTGSP crystals has been investigated under pyroelectric effect conditions. It has been established that in doped crystals there is observed a reproducible emission effect. An equation for the depolarizing field determining the exoemission current has been derived. It is shown that in crystals with fixed domain structure near the Curie point the emission kinetics is limited by the temperature behaviour of the ratio $\gamma / \varepsilon$ (where $\gamma$ is the pyroelectric coefficient, $\varepsilon$ is the dielectric constant).
\end{abstract}

In a single-domain ferroelectric any scalar or vector action brings about a change in the initial charge compensation and the emergence of a depolarizing field. In case of a free surface such an electrostatic field causes a tunnel electronic emission from the polar cut of the crystal. Exoelectron emission has been observed under conditions of pyro-, piezo- and anomalous photovoltaic effects, as well as with switching over of spontaneous polarization. ${ }^{1-6}$ The revealed emission effect allows one to investigate the dynamics of the formation and relaxation of the depolarizing field. Of special interest is the region of ferroelectric phase transition for which the pyroelectric coefficient $\gamma$ and dielectric constant $\varepsilon$ determining the field parameters have anomalously high values.

The exoemission effect in the region of the ferroelectric phase transition has been investigated in detail for non-doped crystals TGS, ${ }^{2,7-10}$ which are characterized by a mobile domain structure. In References 7 and 8 the exoemission has been observed over the range of $290-340 \mathrm{~K}$. The most interesting fact is that the emission curves for different samples did not coincide, but the plotted averaged exoemission curve exhibits two maxima: one in the region below $T_{c}$, the second 2 to $3^{\circ} \mathrm{K}$ above $T_{c}$. The authors think that the first maximum is connected with the restructuring of the domain structure, while the second is associated with its disappearance in the proximity of the Curie temperature. Together with exoemission a domain structure has been observed in TGS with the help of liquid crystals. ${ }^{8}$ The movement of domain boundaries long before the transition into the paraphase beginning from $300 \mathrm{~K}$ has been established. The obtained results are practically identical to data of References 9 and 10 , where two emission peaks have also been observed. It should be noted that in Reference 9 two samples have been investigated the current dependences $I_{e}(T)$ of which do not coincide. The emission current density was $10^{-12}$ A.m ${ }^{-2}$. We investigated single crystals ADTGSP grown in a deuteriumized aqueous solution containing $10 \mathrm{~mol}$. $\% \mathrm{~L}$ - $\alpha$-alanine. The content of orthophosphoric 
acid $\mathrm{H}_{3} \mathrm{PO}_{4}$ was varied in the range of $10-50 \mathrm{~mol} . \%$. The growth temperature was $55^{\circ} \mathrm{C}$, the supersaturation of the solution being $(1.3 \pm 0.2) 10^{-3}$. The action of the ligands alanine and deuterium is known. It leads to the creation of a rigid domain structure. The doping with phosphorus has been studied little. But in Reference 11 it has been noted that the replacement of the sulphate group by the phosphate group in TGS raises the pyroelectric coefficient twice, leaving the dielectric constant unchanged. Samples measuring $5 \times 5 \times 0.5 \mathrm{~mm}^{3}$ were prepared. The large face was perpendicular to the polar axis.

The emission current measurements were conducted in a chevron microchannel electron multiplier set-up under vacuum of $10^{-5}$ Torr. The crystal was in direct contact with the input plane of the first multiplier. The gap between the electron multiplier and the sample corresponded to the surface roughness and was equal to approximately $5 \mu \mathrm{m}$. The ratio $\gamma / \varepsilon$ was measured also.

Figures 1 and 2 show the temperature dependences of the emission current and of the ratio $\gamma / \varepsilon$ for samples having different levels of doping with phosphorus. The heating rate was $\alpha=510^{-2} \mathrm{~K} \cdot \mathrm{s}^{-1}$.

The emission current was registered from the polar $Y$-cuts. As can be seen from Figure 1 curve $I_{e}(T)$ has the form of a wide maximum starting almost immediately with the rise of temperature and trailing, depending on the concentration of the ligand, to $T \sim 343-351 \mathrm{~K}$.

The highest current density in the maximum has been registered for an ADTGSP crystal $\left(10 \mathrm{~mol} . \% \mathrm{H}_{3} \mathrm{PO}_{4}\right) I_{e \text { max }}=6.510^{-9} \mathrm{~A} . \mathrm{m}^{-2}$, this being three orders higher than the emission current density in pure TGS in Reference 9. It is important to note that the results for samples of the same composition were reproducible. The temperature corresponding to the maximum value of the emission current $T_{\max }$ is

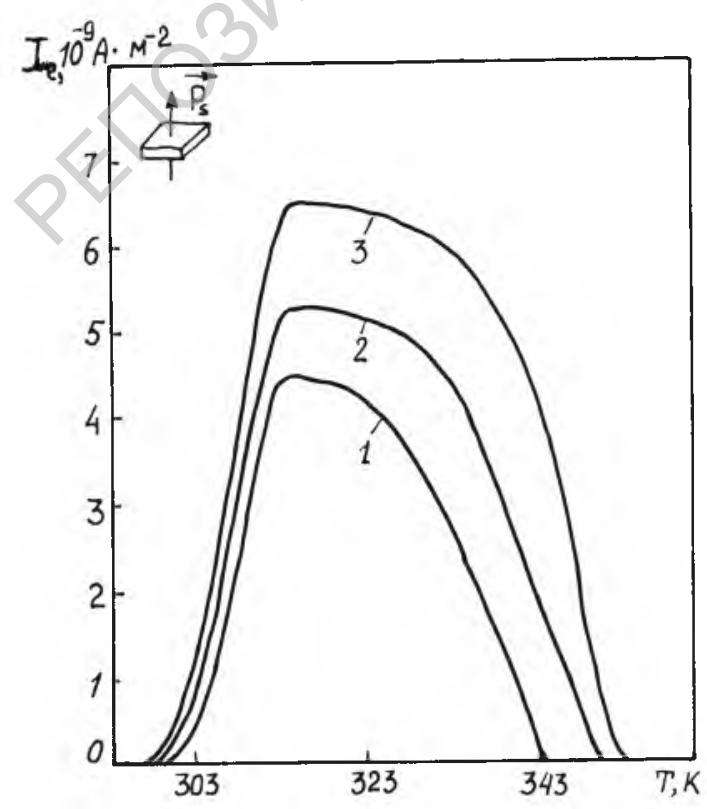

FIGURE 1 Temperature dependence of exoemission from the polar cut of ADTGSP single crystals grown in solutions of $50-1,30-2$, and $10 \mathrm{~mol} \% \mathrm{H}_{3} \mathrm{PO}_{4}-3$. (Growth temperature $55 \mathrm{C}$ supersaturation $(1.3 \pm 0.2) \cdot 10^{-3}$, growth pyramid $\left.(001)\right)$. 


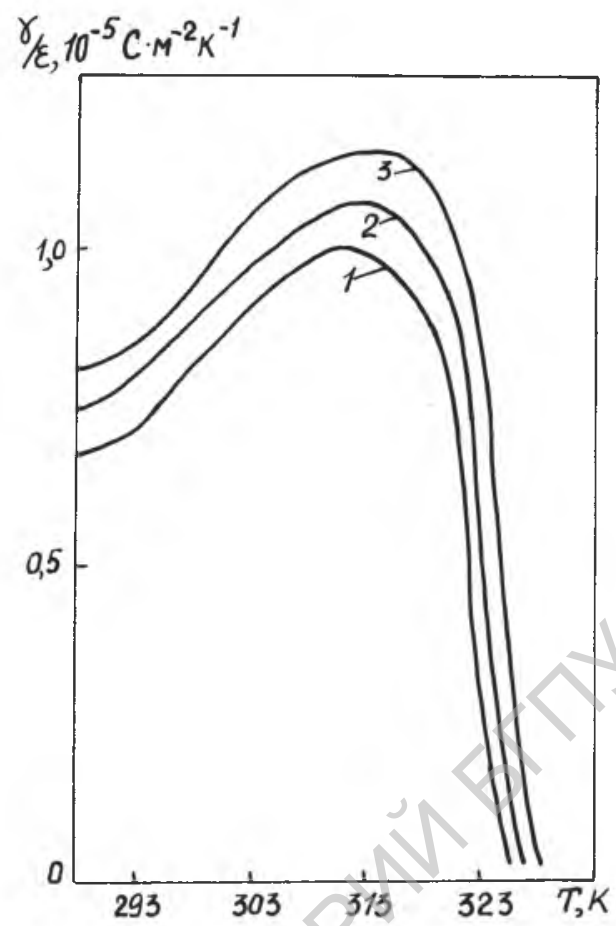

FIGURE 2 Temperature dependence of parameter $\gamma / \varepsilon$ of ADTGSP single crystals grown in solution of $50-1,30-2$, and $10 \mathrm{~mol} \% \mathrm{H}_{3} \mathrm{PO}_{4}-3$. (Growth temperature $55 \mathrm{C}$, supersaturation $(1.3 \pm 0.2) \cdot 10^{-3}$, growth pyramid (001)).

within $311-315 \mathrm{~K}$. The measurement of the temperature dependence $\varepsilon(T)$ has shown the phase transition temperature for these crystals is within $329-331 \mathrm{~K}$, that is, $T_{\max }<T_{c}$.

At the same time one observes practically complete correspondence in the temperature behaviour of the curves $I_{e}(T)$ and $\gamma / \varepsilon(T)$. For example, the temperatures of the maxima for the functions $I_{e}(T)$ and $\gamma / \varepsilon(T)$ coincide, as well as the temperatures at which the functions dependences begin to droop sharply. If the content of $\mathrm{H}_{3} \mathrm{PO}_{4}$ diminishes from $50 \mathrm{~mol} . \%$ to $10 \mathrm{~mol} . \%$, the ratio $\gamma / \varepsilon$ increases (Figure 2). The emission current grows in a similar manner at the same temperatures (Figure 1). The obtained correlations indicate that there exists a functional connection between exoemission current density and the parameter $\gamma / \varepsilon$.

Let us examine a simplified typical scheme of an exoemission experiment (Figure 3 ). The ferroelectric crystal with thickness $d$ in the direction of the polar axis is parallel to the input plane of the electron detector at a distance $a$. For purposes of registration of exoemission the bottom polar face of the crystal and the input plane of the detector are grounded (are at zero potential). Then for the central points of the crystal the potential drop $\varphi_{a}$ in the field of the uncompensated pyroelectric charge $\Delta \rho$ in the crystal-detector space is equal to the potential drop $\varphi_{d}$ over the crystal, that is, $\varphi_{a}=\varphi_{d}$, or, considering the field to be homogeneous

$$
E_{d} \cdot d=E_{a} \cdot a
$$

In (1) $E_{a}$ and $E_{d}$ are intensities of the depolarizing fields in the crystal-detector 


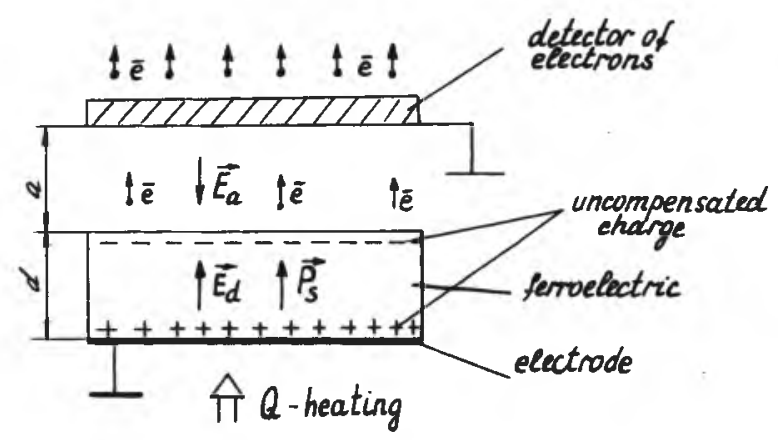

FIGURE 3 The scheme of the exoemission experiment.

space and in the crystal, respectively (the source of fields $E_{a}$ and $E_{d}$ is the charge $\Delta \rho)$.

It is known that when passing across a surface, carrying electrical charges, the normal component of the electrostatic induction vector experiences a jump equal to the surface charge density. Then

$$
\varepsilon_{o} E_{a}+\varepsilon \varepsilon_{o} E_{d}=\Delta \rho
$$

In Reference 12 basing on the sign of the emitting surface charge and the specific scattering mechanisms it is shown that the exoemission effect of ferroelectrics is determined by the depolarizing field $E_{a}$.

The joint solution of (1) and (2), with the pyroelectric effect equation $\Delta \rho=$ $\gamma \Delta T$ taken into consideration, yields

$$
E_{a}=\frac{\gamma \Delta T}{\varepsilon_{o}} \cdot \frac{1}{\varepsilon \frac{a}{d}+1}
$$

In expression (3) the relaxation processes are ignored. It is known that the minimization of the depolarizing field can proceed in two ways: by breaking up into domains and screening. In TGS both types of relaxation processes can proceed depending on the mobility of the domain structure.

A strong domain instability is characteristic of non-doped crystals TGS. The measurements of the thermal Barkhausen effect ${ }^{13}$ show the break-up of singledomain state in pure TGS with temperature change. The maximum of "thermal" repolarization jumps is observed at a temperature several degrees below $T_{c}$. Precision measurements of the pyroelectric coefficient for undoped TGS have shown that the dependence $\gamma(T)$ has a random character and for different samples does not coincide. ${ }^{14}$ Considering expression (3) it is clear that the dependence $E_{a}(T)$ will also have a random character. This explains the unreproducibility of exoemission data obtained on non-doped crystals TGS in References 7-10. The formation of multidomain structure leads to the reduction of the depolarizing field and a low emission current density.

The doping of TGS crystals with additives and, especially, $L$ - $\alpha$-alanine fixes the domain structure precluding the break-up of single-domain state. In this case it is necessary to take into consideration the depolarizing field screening processes. In 
Reference 15 the following relationship for charge $\Delta \rho$, with screening taken into account, has been obtained:

$$
\Delta \rho=\frac{\gamma \alpha}{K}(1-\exp (-k t))
$$

where $t$ is the time, $k$ is the screening rate.

In vacuum one can ignore the leakage of charges from the surrounding atmosphere and consider that the screening of field $E_{a}$ takes place with Maxwell's relaxation time $\tau_{M}$, that is, $k=1 / \tau_{M}$, where $\tau_{M}=\varepsilon \varepsilon_{o} / \delta$ ( $\delta$ is the conductivity). Remembering that in case of a linear change in temperature $T=\alpha \cdot T$ (4) can be expressed as follows:

$$
\Delta \rho=\gamma \alpha \tau_{M}\left(1-\exp \left(-\frac{\Delta \tau}{\alpha \tau_{M}}\right)\right)
$$

Expanding the exponent in a series and limiting the series to three terms, we have a formula for field $E_{a}$ :

$$
E_{a}=\frac{\gamma \Delta T}{\varepsilon_{o}} \frac{1}{\varepsilon \frac{a}{d}+1}\left(1-\frac{\Delta T}{2 a \tau_{M}}\right)
$$

Expression (6) shows that field $E_{a}$ determining the emission current depends on the main physical characteristics of the polar crystal: pyroelectric coefficient, dielectric constant and conductivity. At temperatures remote from the Curie point $\varepsilon$ $=$ const and, as has been shown above, the emission current kinetics for lithium tantalate ${ }^{16}$ is determined by the temperature behaviour of $\gamma$ and $\delta$. Near the Curie point $\varepsilon=\varepsilon(T)$ and formally it follows from (6) that for crystals at these temperatures $E_{a}=E_{a}(\gamma(T), \varepsilon(T), \delta(T))$. The dependence of field $E_{a}$ on conductivity $\delta(T)$ is taken into account in the term $\Delta T / 2 \alpha \tau_{M}$ which, evidently, accounts for the screening of the depolarizing field. According to Reference 17 the value of the dielectric constant for these crystals varies over the range $\varepsilon(T=308 \mathrm{~K}) \sim 10^{2}$ to $\varepsilon\left(T=T_{c}\right) \sim 10^{4}$. For $\alpha=510^{-2} \mathrm{~K} \cdot \mathrm{s}^{-1}$ and $\delta \sim 10^{-13} \mathrm{Ohm}^{-1} \mathrm{~cm}^{-1} 17$ the value of the characteristic temperature $\alpha \tau_{M}$ will lie within $2 \alpha \tau_{M}=\left(10-10^{3}\right) \mathrm{K}$, that is, one can ignore the effect of screening on the depolarizing field and exoemission effect in crystals with fixed domain structure and sufficiently low conductivity in the region of the phase transition due to a sharp rise of $\varepsilon$. Besides, for $\varepsilon>10^{2}, a$ $=510^{-4} \mathrm{~cm}, d=5 \cdot 10^{-2} \mathrm{~cm}, \varepsilon(T) a / d>1$. Expression (6) can then be simplified:

$$
E_{a}=\frac{\gamma(T) \Delta T}{\varepsilon_{o} \varepsilon(T) \frac{a}{d}}
$$

It is seen from (7) that $E_{a}$ is proportional to the ratio $\gamma / \varepsilon$. Since the emission current density is determined by the intensity of the depolarizing field, ${ }^{12}$ it is clear that the exoemission kinetics is limited by the temperature behaviour of parameter $\gamma / \varepsilon$, this being observed experimentally (Figures 1,2 ). The stable single-domain state in doped TGS crystals presets the determined behaviour of $\gamma / \varepsilon$ and $I_{e}(T)$.

Besides this, as it follows from the data presented in Figures 1 and 2 the emission 
current trails to $343-351 \mathrm{~K}$, while the value of $\gamma / \varepsilon \rightarrow 0$ at $T \sim 330 \mathrm{~K}$, corresponding to the temperature of the maximum dielectric constant. Most probably the observed effect is associated with the finite relaxation time of the charge and field on the free surface. This effect does not exhibit itself on crystals with electrodes, that is, under conditions when the parameter $\gamma / \varepsilon$ is registered. For checking this hypothesis the time dependences of emission current relaxation $\tau_{\eta}$ were registered at constant temperature. At $T=323 \mathrm{~K}$ the values of $\tau_{\eta}$ for the investigated samples grown in a solution with 10,30 and $50 \mathrm{~mol} . \%$ were 210,120 and $80 s$, respectively. Under conditions of constant temperature change rate $\alpha=5 \cdot 10^{-2} \mathrm{~K} \cdot \mathrm{s}^{-1}$ the emission current will trail for the given crystals by $10.5,6$ and $4 \mathrm{~K}$, respectively. These values are somewhat less than the experimentally observed (Figure 1).

Thus, measurements of exoemission make it possible to determine the stability of the single-domain state and to evaluate the quality of a pyroelectric material.

\section{REFERENCES}

1. B. Rosenblum, P. Bräunlich and J. Carrico, J. Appl. Phys. Lett., 25-1, 17 (1974).

2. B. Sujak and W. Syslo, Ferroelectrics, 22, 711 (1978).

3. G. I. Rosenman and E. I. Boikova, Phys. St. Sol, A58, k379 (1980).

4. G. I. Rosenman and V. I. Pechorsky, Pisma v JTF, 6-2A, 1531 (1980).

5. G. I. Rosenman, FTT, 30-8, 2323 (1988).

6. G. I. Rosenman, V. A. Okhapkin, Yu. L. Chepelev and V. Ya. Shur, Pisma vJETF, 39-9, 397 (1984).

7. K. Biedrzychi, Phys. St. Sol, A93, 503 (1986).

8. K. Biedrzychi and B. Kosturek, Phys. St. Sol, A1C0, 327 (1987).

9. A. M. Kostsov, A. S. Sidorkin, V. S. Zaltsberg and S. P. Gribkov, FTT, 24-11, 3436 (1982).

10. A. S. Sidorkin, A. M. Kostsov and V. S. Zaltsberg, FTT, 27-7, 2200 (1985).

11. A. Bhalla, C. Fang, L. Cross and X. Yao, Ferroelectrics, 54, 151 (1984).

12. G. I. Rosenman, V. I. Pechorskii, Yu. L. Chepelev and E. I. Boikova, Phys. St. Sol, B120, 667 (1983).

13. V. M. Rudiak, Switching Processes in Nonlinear Crystals. Moscow, Nauka, (1986).

14. V. K. Novik, Pyroelectricity of Polar Single Crystals. Doctorate Thesis, Moscow, (1986).

15. G. I. Rosenman, Yu. L. Chepelev, I. S. Rez and N. B. Angert, Kristallografia, 26-3, 616 (1981).

16. G. I. Rosenman, I. S. Rez, Yu. L. Chepelev and N. B. Angert, FTT, 22-11, 3466 (1980).

17. M. S. Tsedrik, S. A. Vasilevsky and B. I. Yanut, In: Ferroelectrics and Piezoelectrics, Kalinin, KGU, 20 (1987). 